death after overdose in elderly female suicide and $12 \%$ for men) and the fourth commonest cause of death by suicide in elderly males. ${ }^{2}$ Interestingly, Purandare et al's findings clearly show that drowning is the second commonest method of suicide by elderly psychiatric patients, with or without a dementia diagnosis.

The diagnosis of drowning itself may be difficult as there are no specific features at autopsy. ${ }^{3}$ During the investigation of a body found in water, a wide range of possibilities other than downing have to be considered: accident, suicide, misadventure or homicide. Even when the diagnosis of drowning is confirmed (or excluded) the manner of death may remain undetermined. ${ }^{3}$ It is also difficult to determine whether underlying natural diseases play a role in the death, wrongly regarded as self-harm. ${ }^{4}$ There are some examples of this; 'sudden death in bathroom' has been reported, ${ }^{5}$ occurring mostly in winter and $80 \%$ of cases were elderly persons who died while bathing. Cardiac arrest and subsequent drowning in bathtubs were attributed to the sudden reduction in blood pressure and cardiac arrhythmia, and not due to fatal self-harm. ${ }^{5}$ Drowning has also been attributed to sudden unexpected death in epilepsy either subsequent to a seizure or occurring suddenly without explanation. ${ }^{6}$ It may be relevant that in a local study in Cheshire, UK, bathroom drowning accounted for $7 \%$ of all elderly drowning. ${ }^{7}$ Niacin deficiency, similar to pellagra, has been reported in high-income countries in people with alcohol dependency with poor eating habits and self-neglect, malabsorption, malignancy and nutritional deficiencies. ${ }^{8}$ It may be reasonable to assume that some elderly people with dementia, especially those who live alone, develop mental and physical changes due to an easily overlooked nicotinamide deficiency which could result in accidental drowning in an attempt to alleviate skin irritation as is the case in some pellagra sufferers. ${ }^{2}$ It is interesting to note that Lunetta et $a l^{3}$ reported that $2.6 \%$ of bodies found in water were found to have died of natural causes after an initially suspected suicide. A review of trends in elderly suicide by drowning in England and Wales 1979-2001 revealed that suicide by drowning in the elderly attracted only $38 \%$ verdicts of suicide but $62 \%$ open verdict. ${ }^{2}$ The high rate of open verdicts in death by drowning compared with any other method of fatal self-harm in England and Wales simply confirms the difficulties in reaching a firm conclusion in drowning death. Combining suicide and all undetermined deaths in suicide by drowning as a matter of course, especially in nationally collected statistics and consequently in research, may result in grossly exaggerated rates and misleading trends. Suicide by drowning is probably not amenable to prevention and although the elderly are often thought to benefit from suicide prevention more than younger adults, this is not likely to be the case regarding drowning, perhaps sadly more so in those with dementia.

1 Purandare N, Oude Voshaar RC, Rodway C, Bickley H, Burns A, Kapur N Suicide in dementia: 9-year national clinical survey in England and Wales. Br J Psychiatry 2009; 194: 175-80.

2 Salib E. Trends in suicide by drowning in the elderly in England and Wales 1979-2001. Int J Geriatr Psychiatry 2005; 19: 175-81.

3 Lunetta P, Smith G, Pentilla A, Sajanntila A. Undetermined drowning. Med Sci Law 2003; 43: 207-14.

4 Byard RW, Houldsworth G, James RA, Gilbert JD. Characteristic features of suicidal drowning: a 20 year study. Am J Forensic Med Pathol 2001; 22: 134-8.

5 Yoshioka N, Chiba T, Yamauchi M, Monma T, Yoshioki K. Forensic consideration of death in the bathtub. Leg Med 2003; 5 (Supp 1): 375-81.

6 Lathers CM, Schraeder PL. Clinical pharmacology: drugs as a benefit and/or risk in sudden unexpected death in epilepsy? J Clin Pharmacol 2002; 42: 123-36.
7 Salib E, Rahim S, El-Nimr G, Habeeb B. Elderly suicide: an analysis of coroner's inquests into two hundred cases in Cheshire 1989-2001. Med Sci Law 2005; 45: 71-80.

8 Kumar P, Clark ML. Nutrition. In Clinical Medicine: 163-4. Baillière Tindall, 1994.

Emad Salib, Peasley Cross Hospital, St Helens WA9 3DA, UK. Email: esalib@hotmail.com

doi: 10.1192/bjp.194.6.562a

Purandare et $a l^{1}$ used National Confidential Inquiry data to compare the characteristics of dementia patients who died by suicide with those of age-matched suicides with other diagnoses. They say empirical data on suicide in dementia are scarce and largely based upon case reports. Although the literature in this area contains a number of interesting, albeit highly atypical, case reports of patients with less common subtypes of dementia who died by suicide, there are also a substantial number of reports on suicide in older adults with various psychiatric diagnoses. The real problem with this literature is the quality of the studies; the majority are methodologically flawed, for example not employing a sensitive method for detecting mild cognitive impairment or absence of a control group, also use of coroner's records or death certificates to determine psychiatric diagnoses, sources known to underreport cases of dementia. The overall finding from this literature is that suicide appears to be uncommon in dementia, although the risk in Huntington's disease is in the region of threefold compared with the general population. ${ }^{2}$ However, in a recent cohort study based on Danish case registers, it was found that for younger patients (50-69 years) diagnosed with dementia during psychiatric hospitalisation the risk of suicide was over eight times that of the age-matched general population, and the risk was threefold for patients aged over $70 .^{3}$

Purandare et $\mathrm{al}^{1}$ report suicide in dementia to be uncommon in the first year following diagnosis and highlight $1-5$ years after first contact with services as the high-risk period. The case-control design of their study used a convenient but not very informative control group, namely age- and gender-matched suicides with other psychiatric disorders, and consequently the findings do not shed much new light on the association between suicide and dementia. No information is provided about severity of dementia or subtype (dementia subtype is of interest since frontotemporal dementia would be expected to be associated with impulsive acts of suicide and self-harm, as frontal lobe impairment is associated with impulsiveness). The finding that suicide is less common soon after diagnosis is counter-intuitive, and contrary to the findings of Erlangsen et $a l^{3}$ where suicide was most common in the first 6 months after diagnosis. There is also evidence that when attempted suicide occurs in dementia it is more common in early, mild disease and when accompanied by depression. ${ }^{4,5}$

1 Purandare $\mathrm{N}$, Oude Voshaar $\mathrm{RC}$ Rodway $\mathrm{C}$ Bickley $\mathrm{H}$, Burns $\mathrm{A}$, Kapur $\mathrm{N}$. Suicide in dementia: 9-year national clinical survey in England and Wales. $\mathrm{Br} \mathrm{J}$ Psychiatry 2009; 194: 175-80.

2 Harris EC, Barraclough, BM. Suicide as an outcome for medical disorders. Medicine 1994; 73: 281-96.

3 Erlangsen A, Zarit SH, Conwell Y. Hospital-diagnosed dementia and suicide: a longitudinal study using prospective, nationwide register data. Am J Geriatr Psychiatry 2008; 16: 220-8.

4 Tsai $\mathrm{CF}$, Tsai SJ, Yang $\mathrm{CH}$, Hwang JP. Chinese demented inpatients admitted following a suicide attempt: a case series. Int J Geriatr Psychiatry 2007; 22: 1106-9. 
5 Osvath P, Kovacs A, Voros V, Fekete S. Risk factors of attempted suicide in the elderly: the role of cognitive impairment. Int J Psychiatry Clin Practice 2005; 9: 221-5.

Camilla Haw, St Andrew's Healthcare, Billing Road, Northampton NN1 5DG. Email: chaw@standrew.co.uk

doi: 10.1192/bjp.194.6.563

Authors' reply: We welcome the interest in our study of suicide in patients with dementia in England and Wales. We found relatively lower risk of suicide during the first year of illness in dementia. Dr Haw writes that our findings are contrary to findings by Erlangsen et al. ${ }^{1}$ However, such comparison is inaccurate. Erlangsen et al compared the risk of suicide in patients who were diagnosed with dementia during hospitalisation for physical or psychiatric illness with the risk of suicide in the general population. The authors point out that 'the findings cannot be generalised to persons with dementia who have not received the diagnosis while hospitalised'. The risk of suicide is known to be increased around the time of psychiatric hospitalisation. ${ }^{2}$ Psychiatric in-patients would be expected to have more psychiatric disturbances. The study by Tsai et $a l^{3}{ }^{3}$ which Haw quotes to support the association with mild dementia, found that delusions were present in all seven of those who later died by suicide. Haw also seems to compare the literature on increased risk of attempted suicide in those with mild cognitive impairment with our study of completed suicide in patients with diagnosed dementia. One consideration during disclosure of the diagnosis of dementia is the potential for adverse reactions. Our findings suggest that unless the risk assessment, which should be done in any patient being given a diagnosis of a major physical or mental illness, identifies a specific suicide risk, the 'fear of suicide' should not be a major factor in the decision to not disclose the diagnosis of dementia.

We thank Salib who correctly points out that our Method omitted ICD-9 which was indeed the classification system in use by the Office of National Statistics in the earlier part of the study. The relevant ICD-9 codes were E950-E959 and E980-989 (excluding E988.8).

Our findings are based on National Confidential Inquiry data, so include individuals who died by suicide within 12 months of contact with specialist health services. When we examined general population deaths (suicide and undetermined verdicts) in older people during the period covered by this study, drowning was the third most common method of suicide overall after hanging and selfpoisoning (National Confidential Inquiry into Suicide and Homicide, personal communication, 2009). This is consistent with Salib's findings. We agree that the method of suicide may be an important determinant of verdict and there are difficulties in establishing suicide as a cause in drowning. However, this does not affect our main findings, which are based on the conventional definitions of suicide used in previous research and national statistics.

Suicide prevention requires a variety of strategies. ${ }^{4}$ Although we agree that restricting access to drowning as a method of suicide may not be feasible, we do not agree that suicide prevention is futile in this group. Other strategies, for example the improved assessment and treatment of mental disorders, are likely to be worthwhile. We do not accept that younger individuals may be less amenable to prevention. However, different age groups may require a different preventive emphasis. ${ }^{5}$

1 Erlangsen A, Zarit SH, Conwell Y. Hospital-diagnosed dementia and suicide: a longitudinal study using prospective, nationwide register data. Am J Geriatr Psychiatry 2008; 16: 220-8.

2 Qin P. Nordentoft M. Suicide risk in relation to psychiatric hospitalization: evidence based on longitudinal registers. Arch Gen Psychiatry 2005; 62: 427-32
3 Tsai CF, Tsai SJ, Yang $\mathrm{CH}$, Hwang JP. Chinese demented inpatients admitted following a suicide attempt: a case series. Int J Geriatr Psychiatry 2007; 22: 1106-9.

4 Department of Health. National Suicide Prevention Strategy for England. Department of Health, 2002.

5 Hunt IM, Kapur N, Robinson J, Shaw J, Flynn S, Bailey H, et al. Suicide within 12 months of mental health service contact in different age and diagnostic groups: national clinical survey. Br J Psychiatry 2006; 188: 135-42.

Nitin Purandare, Psychiatry Research Group, School of Community Based Medicine, The University of Manchester, Room 3.319, University Place (3rd Floor East), Oxford Road, Manchester M13 9PL. Email: nitin.purandare@manchester.ac.uk; Richard C. Oude Voshaar, University of Manchester, Psychiatry Research Group, School of Community Based Medicine, UK, and Radboud University Nijmegen Medica Centre, Department of Psychiatry, Nijmegen, The Netherlands; Cathryn Rodway, Harriet Bickley, University of Manchester, Centre for Suicide Prevention, UK; Alistair Burns, University of Manchester, Psychiatry Research Group, School of Alistair Burns, University of Manchester, Psychiatry Research Group, School of
Community Based Medicine, UK; Nav Kapur, University of Manchester, Centre for Suicide Prevention, UK.

doi: 10.1192/bjp.194.6.564

\section{Adolescent-onset anorexia nervosa - missing half of the story?}

As a psychiatrist working in an eating disorder service, I am always intrigued by the stability of the eating disorder diagnosis over time. As quoted by Treasure et al, ${ }^{1}$ 'when long-term prognosis is considered, the overlap between anorexia nervosa and bulimia nervosa becomes more striking. We all know that patients diagnosed with anorexia later switch over to other eating disorders and vice versa. ${ }^{2}$ Hence, the paper about long-term outcome of anorexia nervosa ${ }^{3}$ attracted my attention. The methodology of recruiting is vital in a population-based study. Wentz et al have taken extreme steps to be as rigorous as possible. But I consider that they might have overlooked some of the aspects. The authors have described how the diagnosis of individual patients has changed over time from anorexia nervosa to bulimia nervosa to no eating disorder to eating disorder not otherwise specified (EDNOS) (their Fig.1). This highlights the diagnostic instability of these groups of illnesses. The authors have assessed individuals cross-sectionally and asserted them to have anorexia nervosa. The important information missing here is whether these individuals had symptoms of other eating disorders such as EDNOS before having symptoms of anorexia. Since the study is about long-term outcome of anorexia nervosa, Wentz et al should have taken adequate care to ascertain that the cohort they were following did in fact belong to the anorexia nervosa group. This drawback is further highlighted in the exclusion criteria of the study. Excluding patients at the initial stage (Study 1 ) of individuals with a history of eating disturbances could have excluded individuals who might have been suffering from a non-anorexic type of eating disorder. The authors assert that in the subsequent study they did not exclude patients who crossed over to other eating disorders (thereby promptly registering changes prospectively), but what they did by excluding certain people is to exclude these potential participants who could have shown crossing over from another type of eating disorder to anorexia nervosa.

There are other minor points that are worth mentioning. Comprehensive screening, that would have included patients of all severity, of individuals who were born in a particular year (1970), identified 24 cases of anorexia nervosa. Combining this with a less comprehensively assessed group of individuals (and thereby potentially picking up only very severe cases) could have resulted in heterogeneous populations being mixed. Instead of mixing these two cohorts with potential difference in their severity with a possible impact over their outcome including complications, ${ }^{4}$ the authors could have treated them as two 